\title{
Enden der Liebe, Enden des Texts
}

\author{
Der Alltag der Liebe bei Navid Kermani und \\ Karl Ove Knausgård
}

\begin{abstract}
Der in den 1970er Jahren von Serge Doubrovsky geprägte Begriff der Autofiktion ist in den letzten Jahren zu einem Modell aufgestiegen, das den Kunstund Literaturbetrieb dominiert. Sein Konzept der Autofiktion will nicht auf die großen Momente eines wirkungsmächtigen Lebens zurückblicken, sondern das gegenwärtige Alltagsleben in eine literarische Form bringen. Spricht man über das Private, ist die Liebe nicht fern, denn es gibt nichts Privateres. Die Liebe ist sogar ausschließlich persönlich, da jeder, der über die Liebe spricht, von seinen spezifischen und individuellen Erfahrungen ausgeht. Die Darstellung der Liebe in den autofiktiven Romanen von Navid Kermani und Karl Ove Knausgård zeigt die Problematik der Grenzen des Privaten und die mit der Buchveröffentlichung einhergehende literarische Appropriation der Partnerin auf. Die Literatur ist in beiden hier vorgestellten literarischen Großprojekten ein Medium, in dem man weiter gehen kann als in der zwischenmenschlichen Kommunikation des Alltags. Kermani greift in letzter Konsequenz mit einem literarischen Kunstgriff zur Autozensur, während Knausgård behauptet, mit Ende des Romans seine literarischen Ambitionen zu beenden. Die Autobiographie endet also nicht mit dem Tod, sondern mit dem Tod des Autors.
\end{abstract}

Keywords: Autofiktion; Navid Kermani; Karl Ove Knausgård; Liebe; Tonje Aursland; Literatur; Dein Name; Min Kamp

\section{Hunger nach Realität}

Alles, was uns ausmacht, beginnt mit $L$ wie Literatur: Luft, Lust, Liebe, Lesen, Leib und Leben. Ein L sollte noch gehen: Ludwig. Wittgenstein also. Dieser wünschte in einer Manuskripteintragung vom 22. August 1930 zum Voyeur zu werden:

Es könnte nichts merkwürdiger sein, als einen Menschen bei irgend einer ganz einfach alltäglichen Tätigkeit, wenn er sich unbeobachtet glaubt, zu sehen. Denken wir uns ein Theater, der Vorhang ginge auf und wir sähen einen Menschen allein in seinem Zimmer auf und ab gehen, sich eine Zigarette anzünden, sich niedersetzen, u.s.f., so, daß wir plötzlich von außen einen Menschen sehen, wie man sich sonst nie sehen kann; wenn wir quasi ein Kapitel einer Biographie mit eigenen Augen sehen, - das müßte unheimlich und wunderbar 
zugleich sein. Wunderbarer als irgend etwas was ein Dichter auf der Bühne spielen oder sprechen lassen könnte, wir würden das Leben selbst sehen. (Wittgenstein 2015, 456)

Das 21. Jahrhundert verwirklichte Wittgensteins Überlegungen radikaler, als sich dieser das je hätte vorstellen können. Kein Fernsehsender kommt ohne selbstproduzierte „Reality Show“ aus, in denen man Menschen sieht, welche die ihnen zustehenden „fifteen minutes of fame“ abholen, angehende Models oder Musiker, die man bei der Verrichtung ihres Alltags betrachten kann. Diese Form der Wirklichkeitsbeobachtung, bei der man durch (kostenpflichtiges) Voting den Verlauf der Show mitbestimmen kann, ist so populär, dass im Jahr 2008 mehr Stimmen für „American Idol“ abgegeben wurden als für Barack Obama bei der Präsidentschaftswahl (vgl. Shields 2010, Nr. 303). ${ }^{1}$ Der Realität der täglich uns überschwemmenden Katastrophenmeldungen halten wir anscheinend nicht Fiktion entgegen, sondern reale Ereignisse im Reagenzglas, laut David Shields deshalb, weil wir selbst kaum noch Realität erleben (vgl. Shields 2010, Nr. 242), absurderweise genau deshalb, weil sich die Freizeit zu oft darauf beschränkt, das Leben der Anderen im digitalen Raum zu verfolgen. Die überzeugendsten kreativen Energien scheinen heute in den nichtfiktionalen Bereich zu fließen, überlegt Shields und hält fest: „Biography and autobiography are the lifeblood of art right now“ (Shields 2010, Nr. 67). Und tatsächlich boomt am Buchmarkt länder- und sprachübergreifend das Spiel mit der Autofiktion.

\section{Autofiktion}

Der in den 1970er Jahren von Serge Doubrovsky geprägte Begriff der Autofiktion ist in den letzten Jahren zu einem Modell aufgestiegen, das den Kunst- und Literaturbetrieb dominiert. Der Begriff ist unscharf definiert und wird in der Regel als Hybrid zwischen Roman und Autobiographie gesehen: Autor, Erzähler und Protagonist sind ident, die Texte jedoch als Roman gekennzeichnet.

Ein Problem der traditionellen Autobiographie ist, dass diese Gattungsbezeichnung einschüchtert, da man an die Werke von Augustinus, Rousseau oder Goethe erinnert wird. Dies bestätigt auch Serge Doubrovsky: „Autobiographie? Non, c'est un privilège réservé aux importants de ce monde, au soir de leur vie, et dans un beau style“ (Doubrovsky 1977, Paratext auf der Rückseite des Umschlags). Sein Konzept der Autofiktion will dagegen gar nicht auf die großen Momente eines wirkungsmächtigen Lebens zurückblicken, sondern das gegenwärtige Alltags-

197 Millionen Stimmen für „American Idol“ gegen 70 Millionen für Obama. 
leben in eine literarische Form bringen. So ist es - neben der Genrebezeichnung „Roman“ - vor allem die Form, die für Doubrovsky das fiktionale Element seiner Autofiktion ausmacht. ${ }^{2}$

Viele Texte der Autofiktion gehen allerdings weiter und spielen nicht nur formal, sondern auch inhaltlich mit den Möglichkeiten des Romans und bieten dem Leser neben dem „pacte autobiographique“ auch einen „pacte romanesque“ (Darrieussecq 1996, 7) an. Der „pacte romanesque“ ist das kulturelle Einverständnis, dass Schriftsteller sich von der Wahrheit entfernen dürfen, ohne dass ihnen vorgeworfen wird, zu lügen. Obwohl man also weiß, dass Romane eine nicht-wirkliche Geschichte erzählen, wird ihnen trotzdem geglaubt (vgl. Zipfel 2009). Autofiktionale Bücher weichen analog dazu ebenso von der Wahrheit ab, ohne ihren Wahrheitsanspruch aufzugeben. „Überhaupt erfinde ich als Romanschreiber nur dort, wo es der Wahrheitsfindung dient“ (Kermani 2016, 30), schreibt Navid Kermani in seinem neuesten Roman. Durch diese wechselseitige Befruchtung von Fakt und Fiktion begibt sich der Erzähler durchaus in die Nähe von postfaktischen Narrationen oder ,alternative facts“ (Hoffmann 2018, 16).

Die Autofiktion wird dadurch viel mehr als eine Spielart der Autobiographie, wie man gleich anhand der gewählten Beispiele sehen wird. Das Subjekt konstituiert sich darin erst durch das Schreiben, wodurch sich das Schreiben unablässig selbst reflektiert. Folglich wird die Autofiktion zu einer Meta-Autofiktion, in der weniger die Erlebnisse des Lebens abgehandelt werden, als dass das Schreiben und das Erinnern in den Vordergrund rücken. Dadurch verliert die der Vergangenheit angehörende Lebensgeschichte an Bedeutung und die Gegenwart rückt in den Fokus. Die zentrale Frage ist, was das Erinnerte dem schreibenden Subjekt zum Zeitpunkt der Niederschrift bedeutet (vgl. Ott und Weiser 2013, 9). Der Zeitpunkt der Niederschrift wird deshalb wesentlicher Teil des Texts und nicht von ungefähr beginnen sowohl Navid Kermani als auch Karl Ove Knausgård ihre autobiographischen Romane mit einer Zeitangabe. Kermanis erster Satz lautet: „Es ist Donnerstag, der 8. Juni 2006, 11:23 auf dem Laptop...“ (Kermani 2011, 7). Knausgård hebt im letzten Band seiner sechsbändigen Romanbiographie folgendermaßen an: „I midten av september 2009 dro jeg opp til Thomas og Maries lille landsted...“. (Knausgård 2011, 7)

2 Es ist bezeichnend, dass auch die Bezeichnung „autofiction“ zuerst in einem Paratext vorkommt: „Fiction, d'événements et de faits strictement réels; si l'on veut, autofiction, d'avoir confié le langage d'une aventure à l'aventure du langage, hors sagesse et hors syntaxe du roman, traditionnel ou nouveau.“ (Doubrovsky 1977, Paratext auf der Rückseite des Umschlags).

3 „Mitte September 2009 fuhr ich rauf zu Thomas und Maries kleinem Landhaus.... " [meine Übersetzung, S.K.]. 


\section{Der Alltag}

Das gesamte Min Kamp betitelte autobiographische Projekt Knausgårds umfasst 3600 Seiten, die in Norwegen zwischen 2009 und 2011 erschienen, zu heftigen Diskussionen über die Grenzen des Privaten Anlass gaben und zu einem der größten Verkaufserfolge des norwegischen Buchhandels überhaupt wurden. Aber auch international wurde Knausgård zu einem „global rockstar“ ausgerufen (The Economist) und als „Proust norvegese“ (La Stampa) gefeiert.

Wie kein Held der Literaturgeschichte zuvor geht Knausgård alleine auf und $\mathrm{ab}$, zündet sich eine Zigarette an und setzt sich nieder, genauso wie im imaginären Theater Wittgensteins vorweggenommen. Hier kann man tatsächlich den Alltag in seiner alltäglichsten Form sehen:

(Jeg) hentet de to avisene som lå på gulvet foran utgangsdøren, satte på kaffetrakteren, leste kulturen og sporten og spiste et eple mens jeg ventet på at kaffen skulle bli ferdig. Da den var det, drakk jeg en kopp og røykte en sigarett ute på verandaen. Himmelen var disig, demringsmørkets grå svevde fortsatt mellom bygningene under meg, og hadde noe rått ved seg; det var midten av august og snart høst.

Jeg tente en sigarett til for å trekke ut stunden før arbeidet begynte sålenge som mulig, men stumpet den halvrøykt og gikk inn på kontoret, skrudde på pc-en, satte meg ned, tente lampen som var festet med en klype i bokhyllen, bladde gjennom stabelen med cd-er so stod på gulvet ved siden av, bestimte meg fro boo Radleys' Giant Steps... (Knausgård 2011, 84) ${ }^{4}$

In Navid Kermanis ebenfalls 2011 erschienenem autobiographischen Roman Dein Name wird weniger geraucht, aber auch in diesem 1200 Seiten starken Buch versucht der Autor so ehrlich wie möglich seinen Alltag - auch hier den Alltag eines Familienvaters und Schriftstellers - in der Literatur abzubilden. Während die Kontroverse um Knausgårds Bücher in Norwegen auch deshalb so hohe Wellen

\footnotetext{
4 „Ich nahm die zwei Zeitungen, die am Boden vor unserer Tür lagen, schaltete die Kaffeemaschine ein, las den Kulturteil und den Sportteil und aß einen Apfel, während ich darauf wartete, dass der Kaffee fertig wurde. Als er fertig war, trank ich eine Tasse Kaffee und rauchte eine Zigarette draußen am Balkon. Der Himmel war diesig, das Grau der Dämmerung schwebte noch immer zwischen den Gebäuden unter mir, die auch etwas Graues hatten. Es war Mitte August und bald Herbst.

Ich zündete eine weitere Zigarette an, um den Augenblick, an dem ich drinnen zu arbeiten beginnen musste, so lange wie möglich hinauszuschieben, drückte aber dann doch schon die nur halbgerauchte Zigarette aus und ging hinein ins Büro, schaltete den Computer ein, setzte mich, schaltete die Lampe ein, die mit einer Klemme auf dem Bücherregal befestigt war, blätterte im Stapel CDs, der am Boden auf meiner Seite stand, entschied mich für Boo Radleys Giant Steps... “ [meine Übersetzung, S.K.].
} 
schlug, weil der Schriftsteller alle beteiligten Personen mit realen Namen nennt, ${ }^{5}$ gibt Kermani in seinem Buch nur diejenigen Namen preis, die im Laufe des Projekts sterben und daraufhin mit einem Nachruf gewürdigt werden. Sich selbst bezeichnet er im Text stets in der dritten Person sprechend als Romanschreiber, Enkel, Sohn, Vater, Mann, Leser, Freund, Nachbar, Liebhaber, Berichterstatter, Orientalist, Handlungsreisender, Nummer zehn, Navid Kermani oder Poetologen (vgl. Kermani 2011, 1065). ${ }^{6}$ Ein Einblick in den Alltag dieses Menschen gibt folgende Szene:

\begin{abstract}
Heute wollte die Familie fahren, aber da die Frau mit ihrem Gips einen unabweisbaren Grund vorwies, verhindert zu sein, bleibt die Familie noch in Köln und schaut das Eröffnungsspiel mit Freunden auf der Terrasse. Er selbst wäre trotz des Heuschnupfens gefahren, um die Tochter zu belügen, daß sie wieder eine Familie sind. Letztes Jahr verschwanden die Pollen, die ihm zusetzen, Anfang oder Mitte Juni von einem auf den anderen Tag, auf den er dieses Jahr also nur warten müßte, um sich wieder aus der Stadt herauszutrauen. Er wollte nur fahren, gesteht er sich ein, damit die Frau wieder nein sagen mußte. Aufs Klo muß er noch, danach stöpselt er das Kabel in die Buchse und lädt ein weiteres Mal die E-Mails herunter. Im Radio läuft Beethoven, Klavierkonzert, das erste, zweite, dritte oder vierte. (Kermani 2011, 9)
\end{abstract}

Der Alltag, das Alltägliche auch hier. Und die Liebe, wenngleich auch die vergehende, die dem romantischen Verliebtsein entgegengesetzte Seite des Liebeszyklus. „Liebe als Passion“ ist als romantisches Konstrukt entlarvt worden (vgl. beispielsweise Luserke-Jaqui 2011), im 21. Jahrhundert bleibt den Dichtern nur noch der Alltag einer Paarbeziehung. Bei Knausgård liegt die Frau noch im Bett, während er um halb fünf Uhr Früh eine Stunde schreibt, bevor die Kinder munter werden, die er dann in den Kindergarten bringen muss, da sich seine Frau, die an einer bipolaren Störung leidet, zu schwach dafür fühlt. Die Krankheit von Kermanis Ehefrau wird nicht so genau benannt: ein Gips, der jedoch auch zu einer Einweisung ins Krankenhaus und psychologischen Problemen führt - in beiden Fällen aber scheint es die kranke und dadurch schwache Frau zu sein, die eine Trennung verhindert.

5 Außer seinen Onkel und ein paar andere Familienmitglieder väterlicherseits, die dagegen protestiert hatten, als ihnen das Manuskript vor Drucklegung vorgelegt wurde, und die deshalb einen Decknamen bekamen.

6 Nur in den 21 Nachrufen, die das Gerüst des Romans darstellen, wechselt Kermani in die erste Person (und in den Szenen, in denen er sich in religiöser oder ästhetischer Ekstase verliert). Selten taucht darüber hinaus ein als „realer Autor“ getarntes „Ich“ auf, das den fiktiven Autor hierarchisch übergeordnet ist und das diesen überstimmen bzw. korrigieren darf (vgl. Hoffmann 2018, 18-19). 


\section{Die Liebe}

Spricht man über das Private, ist die Liebe nicht fern, denn es gibt nichts Privateres (vgl. zu diesem Kapitel auch: Kutzenberger 2019, 411-415). Die Liebe ist sogar ausschließlich persönlich, da jeder, der über die Liebe spricht, von seinen spezifischen und individuellen Erfahrungen ausgeht. Kermani kontrastiert diese Überlegung mit den Fragen nach dem Tod, deren Antworten in der Regel absolut erfahrungslos sind. Es ist noch keiner von uns gestorben, aber geliebt, geliebt haben wir schon alle. Liebe ist daher maximal empirisch (vgl. Kermani 2013).

Will man die Liebe im Privaten belassen, ist es gefährlich, mit einem Schriftsteller verheiratet zu sein, und Tonje Aursland, Karl Ove Knausgårds Ex-Frau, scherzt nicht, wenn sie in einer von ihr selbst produzierten Radiodokumentation sagt, dass es absolute Voraussetzung für ihre nächste Beziehung war, dass ihr neuer Partner nicht schriftstellerische Ambitionen hatte (vgl. Aursland 2010). Navid Kermani erwähnt in seiner Rede zum Kleist-Preis, dass Kleists Penthesilea das brutalste Liebesdrama der deutschen Theatergeschichte ist, in dem die Verliebtheit in Wahnsinn, Tod und Kannibalismus endet. Penthesilea vernichtet Achill, weil sie ihn liebt, sie will ihn ganz und gar in sich aufnehmen, und Kleist lässt keinen Zweifel daran: „Sie hat ihn wirklich aufgegessen, den Achill, vor Liebe“ (Kleist in einem Brief an Marie von Kleist, nach: Kermani 2013, 18). Eine literarische Entsprechung dieses Besitzen-Wollens, dieses ganz und gar in sich Aufnehmens, wäre sicherlich die Appropriation der Geliebten in der Lebenserinnerung. So schwierig es für die Partnerin sein muss, plötzlich gemeinsame Geheimnisse oder intime Gedanken des Ehemanns veröffentlicht zu sehen, so ist dies für Knausgårds Ex-Frau nicht das Verletzendste. Was sie noch schwieriger akzeptieren kann, als in den Kopf des Partners schauen zu müssen, ist vielmehr, dass dieser vorgibt, in den ihren blicken zu können, das heißt, dass er die Handlungen der Partnerin determiniert und durch die Verschriftung zur einzig gültigen macht. Ganz am Ende des fünften Bandes, nachdem Karl Ove Knausgård ihr seine betrunkene Untreue gestanden hat, geht auch Tonje fremd. Sie begründet dies mit ihrer Eifersucht, nicht auf eine Frau, sondern auf die Literatur:

- Hvorfor gjorde du det? Sa jeg. - Jeg var så full at jeg ikke visste hva jeg gjorde. Men du gjør aldri noe som ikke er kontrollert. Du visste hva du gjorde.

- Jeg vet ikke. Jeg tror det var det at du plutselig ble så glad. At du plutselig gikk rundt her og strålte av lykke. Du har vært nedtrykt i fire år, helt siden den høsten faren din døde og du debuterte, og det har vært så tungt, så lite glede. Jeg har forsøkt, jeg har prøvd alt. Og så får du plutselig til å skrive, og så er du glad igjen! (Knausgård 2010, 607)7

7 „Warum hast du es getan?“, sagte ich [d.i. Knausgård]. „Ich war so betrunken, dass ich nicht wusste, was ich tat. Aber du tust nie etwas, das nicht kontrolliert ist. Du hast gewusst, was du 
Auch Kermanis Frau ist eifersüchtig auf die Literatur, sie erkennt, dass ihr Mann immer an den Roman denkt, selbst wenn er intim mit ihr ist:

Und weil auch Gott gern an Schrauben dreht, hat sich die Liebesnot, die der Roman schildert, nicht zuletzt dadurch vertieft, daß der Romanschreiber an den Roman dachte, in dem er die Liebesnot schildert, während er die Frau umarmte, die er noch immer liebt. (Kermani 2011, 1106)

Kermanis Ehe schlittert immer mehr der Scheidung entgegen und Knausgård beschließt, einfach wegzufahren, ohne Schluss zu machen, obwohl seine Ehe für ihn definitiv vorbei ist. Gemeinsam gehen sie zum Bahnhof. „Hun omfavnet meg“, schreibt Knausgård. „Hun gråt“, und als der Zug aus dem Bahnhof rollte, „så jeg Tonje gå alene bortover perrongen o gut i byen.“ (Knausgård 2010, 608) Für Tonje Aursland sind diese Szenen so privat, dass sie nie gedacht hatte, dass sie jemals öffentlich werden könnten. Nur drei Personen wussten bislang, dass sie untreu war, sie, ihr Mann und der Geliebte, und es war ihr fürchterlich peinlich, dass nun ihr Vater, und sogar ihre 90-jährige Großmutter, das durch einen Roman erfahren mussten. Am schmerzhaftesten war aber die Erfahrung, dass Knausgårds Buchfassung durch die Verschriftung plötzlich objektive Wirklichkeit geworden ist: „de er en historieskriving“ (Aursland 2010) ${ }^{9}$, sagt sie in der von ihr produzierten Radiodokumentation, in der sie sich wortwörtlich eine eigene Stimme gibt. Konstanze Fliedl bemerkt sehr ähnlich, Max Frischs Montauk betreffend, dass Frischs Sicht der Dinge zwar subjektiv gewiss aufrichtig sei, letztlich aber doch nur Interpretation des Geschehenen. Durch die Verschriftung werde diese jetzt aber zu „einer Geschichte - ein gedeutetes Faktum“ (Fliedl 2002, 58). Knausgård rechtfertigt sich, dass er ja nichts Schreckliches geschrieben hätte (vgl. Aursland 2010), doch Aursland zeigt auf, dass alleine das Bild der einsam am Bahnsteig zurückgelassenen Ex-Frau sie in die Rolle des Opfers drängen würde, eine Rolle, die sie nicht anzunehmen bereit ist, da sie damals mindestens genauso erleichtert darüber war wie er, dass die Beziehung ein Ende hatte. Nun wird sie allerdings

tust.“ „Ich weiß nicht. Ich glaube, es war, weil du plötzlich so froh warst. Dass du plötzlich herumgingst und vor Glück gestrahlt hast. Vier Jahre lang bist du niedergeschlagen gewesen, seit dem Herbst, in dem dein Vater gestorben ist und du dein Debut veröffentlich hast, und das war so bleiern, so wenig Freude. Und ich habe es versucht, ich habe alles probiert. Und dann läuft es plötzlich beim Schreiben, und schon bist du wieder froh!“ [Meine Übersetzung, S.K.]

8 „Sie umarmte mich“, schreibt Knausgård, „sie weinte“, und als der Zug aus dem Bahnhof rollte, „sah ich Tonje alleine den Bahnsteig hinunter gehen und hinaus in die Stadt.“ [Meine Übersetzung, S.K.]

9 „Das ist Geschichtsschreibung“. [Meine Übersetzung, S.K.] 
für immer die Ex-Frau aus Knausgårds Buch bleiben, ob sie das nun will oder nicht. Marianne Egeland vergleicht diesen Zustand mit totalitären Regimen, denn auch dort dringen Autoritäten in die Privatsphäre ein und bestimmen, was man glauben und denken soll (vgl. Egeland 2015). Aursland akzeptiert das Werk ihres Ex-Mannes freilich als Kunstwerk und beschloss deshalb, als sie das Manuskript vorgelegt bekam, den Text weder zu redigieren noch $\mathrm{zu}$ kommentieren, sondern stattdessen die Radiodokumentation zu machen, in der sie darlegt, wie es sich anfühlt, unfreiwillig eine Romanfigur geworden zu sein.

Für Aursland ist Knausgård „hensynsløs, men med fyktelig daarlig samvittighet“ (Aursland 2010). ${ }^{10}$ Wenn es hart auf hart kommt, würde er immer die Schriftstellerei wählen. Diese Aussage bestätigt Knausgård, wenn er sagt, dass er, wenn sein Bruder von ihm verlangen würde, gewisse Stellen zu streichen, er ohne Zweifel lieber den Bruder verlieren würde. Nur um sich daraufhin zu fragen: „Hvorfor? Var jeg gal?“ (Knausgård 2011, 52) ${ }^{11}$

Auch für Navid Kermani ist es Segen und Fluch des Schriftstellers, dass für diesen die Literatur mehr Wahrheit transportiert als die Wirklichkeit: „Daß die Literatur ihm erlaubte zu sagen, was er von sich nie sagen würde, gehörte zu seinen Privilegien. “(Kermani 2011,13) Die Literatur ist demnach also ein Medium, in dem man weiter gehen kann als in der normalen zwischenmenschlichen Kommunikation. Kermani geht in seiner Autofiktion allerdings ohnehin nicht so weit und führt die Autozensur ein: Am Samstag, den 17. Juni 2006 um 22:08, schreibt er, dass er mit seiner Ehe aufhören wolle.

Was diese Mitteilung für den Roman bedeutet, den ich schreibe, ergibt sich aus dem vorletzten Absatz: Entweder streicht der Mann diesen Absatz, oder er trennt sich tatsächlich, oder die Frau darf den Roman nie lesen, den ich schreibe, jedenfalls nicht die nächsten Jahre. (Kermani 2011, 16)

Was steht aber im vorletzten Absatz?

Wenn er formulierte oder auch nur andeutete, was ihn am Donnerstag, dem 15. Juni 2006, um 16:32 mitteleuropäischer Sommerzeit und schon den ganzen Tag und den Traum davor quält, erhielte der Roman, den ich schreibe, eine andere Beschaffenheit, weil er ihn dann prinzipiell nicht veröffentlichen oder auch nur der Frau zu lesen geben könnte. (Kermani 2011, 13)

Da er es bei dieser Mitteilung belässt, er also weder andeutet noch ausformuliert, was geschehen ist, das eine Veröffentlichung des Romans verhindern würde,

10 „Rücksichtslos, aber mit fürchterlich schlechtem Gewissen.“ [Meine Übersetzung, S.K.]

11 „Warum? War ich verrückt?“ [Meine Übersetzung, S.K.] 
kann man davon ausgehen, dass die Ehe mit seiner Frau nicht geschieden werden musste, da ja auch der Roman veröffentlicht wurde.

Am Ende des Romans lässt sich die Romanfigur Kermani dann allerdings doch von seiner Frau scheiden, was dazu führte, dass vom 5. Juli bis zum 15. August 2012 dem Wikipedia-Eintrag zu Navid Kermani zu entnehmen war, dass sich der Schriftsteller von seiner Frau getrennt hätte (vgl. Hoffmann 2018, 14). Dass der Eintrag wieder gelöscht wurde, könnte als Indiz dafür gelten, dass die Scheidung im Roman für den realen Autor nicht gelten muss: „Meine eigene Geschichte wäre viel banaler, als sie hier steht“, sagt Kermani, ,wenn ein Roman es verlangt, dass sein Protagonist sich scheiden lässt, wird er eben geschieden.“ (Keil 2011) Andererseits beteuert der Erzähler in Dein Name, dass er die Scheidung ,weder auf Erden, noch im Roman gebrauchen kann“ (Kermani 2011, 1080). Ein Indiz für die noch immer bestehende Ehe, so man das literarische Verwirrspiel auflösen will, könnte ein Brief des Schriftstellers an den Manager von Neil Young sein, in dem Kermani schreibt, dass er am 25. Juli 2013 mit seiner Frau und älteren Tochter im Konzert des kanadischen Musikers in Lucca gewesen war und wie sie alle drei engumschlungen in der ersten Reihe gestanden wären, „in love with each other, grateful for the two-and-a-half hours, the fourteen years, and everything that led up to this moment in front of the stage, including even the crises and the pain." (Kermani 2018, 41)

\section{Gelesene Liebe}

Wie hat seine Frau, die deutsch-iranische Islamwissenschaftlerin Katajun Amirpur, um nun wenigsten hier ihren Namen zu nennen, auf das Manuskript reagiert? „Selbst die Frau, die in dem Roman geliebt wird, hat bislang nur den Anfang gelesen, weil der Romanschreiber sich vor ihrer Reaktion fürchtet“ (Kermani 2011, 1108), steht in ebendiesem Roman geschrieben. Als sie schließlich das über 2000 Seiten starke Manuskript vollständig gelesen zurückgibt, erfüllt sich die Hoffnung des Autors nicht, dass sie sein Buch als Liebesbeweis sieht.

\footnotetext{
Ich muss wohl erst tot sein, damit du etwas Nettes über mich sagst. - Herrgott! Rief der Mann, es ist doch ein Roman, den ich schreibe, nicht etwa die Wirklich... und wie es die Wirklichkeit sei, wird sie gedacht haben, als sie die poetologische Debatte mit einer wegwerfenden Geste beendete, ein Abschied wie Sterben, entriß sich, machte eine Bewegung mit der Hand, gleichsam als ,alles sei aus‘. (Kermani 2011, 1196)
}

In dieser Szene wird das Dilemma der radikalen (pseudo-)autobiographischen Fiktion so deutlich wie selten: Einerseits ist das Familienleben, der Alltag einer 
Paarbeziehung, das Kapital jeder Autofiktion, andererseits gilt es, alle darin Beteiligten zu schützen, vor der Öffentlichkeit und vor verletzten Gefühlen.

Als Karl Ove Knausgård seiner zweiten Frau Linda Boström Knausgård Teil 2 von Min Kamp zu lesen gab, bereitete er sie darauf vor, indem er sagte, dass schreckliche Dinge darin stehen würden, dass er es aber nicht böse gemeint hätte. Nach einer Stunde ruft er sie bereits an und fragt, was sie davon halte. „Hun sa at hun syntes det var bra, at det var forferdelig å lese, men at det gikk. Jeg sa at jeg hadde vært frustrert, men at jeg ikke var de nå. Hun sa: farvel, romantikk.“ (Knausgård 2011, 950) ${ }^{12}$ Als sie das Manuskript fertig gelesen hatte, rief sie wieder an und fragte nur weinend: „Hva skal vi gjøre nå, Karl Ove?“ (Knausgård 2011, $952)^{13}$

Es sind aber nicht die großen Fehlleistungen, nicht Untreue oder Lüge, die das Lesen des zu Papier gebrachten Ehealltags für die betroffenen Partnerinnen so schwer machen, es sind die kleinen Ausrutscher und Geheimnisse einer Ehe. Dass Knausgård beim Babyturnen Fantasien mir der hübschen Betreuerin hatte oder dass er der Meinung ist, den Haushalt praktisch im Alleingang zu bewältigen. Die große Untreue findet ohnehin eher in der Fiktion als in der Realität statt. „Wüßte er bereits, daß er einen Roman schreibt, würde er an dieser Stelle eine Affäre erfinden“ (Kermani 2011, 8), schreibt Kermani über einen Besuch in seinem Büro, und tatsächlich findet in seinem Roman Kurzmitteilung (2007) eine Affäre im Büro des Protagonisten statt, in Dein Name dagegen nicht. Knausgård wiederum berichtet in seinem Debutroman Ute av verden (1998) von einer Affäre des Protagonisten, einem jungen Aushilfslehrer, mit einer erst 13-jährigen Schülerin, wogegen im Band IV von Min kamp kurz beschrieben ist, wie ihm als junger Aushilfslehrer eine 13-jährige Schülerin gefallen, er dann aber doch lieber keine Schritte gesetzt hatte.

Um verletzt werden zu können, muss man selbst gar nicht in einem autobiographischen Roman vorkommen. Im zweiten Band von Min Kamp, auf Deutsch Liebe betitelt, kommt Knausgårds erste Frau Tonje kaum vor, weshalb er ihr das Manuskript auch nicht zusandte und sie bat, das Buch nicht zu lesen, da es von seiner Liebe zu seiner zweiten Frau handelt, mit der er zusammengekommen ist, während er noch mit ihr verheiratet war. Umso unvorbereiteter war sie, als Journalisten nach der Veröffentlichung des Buchs anriefen und fragten, wie sie sich bei Stellen wie dieser fühle: „...samtalene med Tonje ble færre og færre. Så

12 „Sie sagte, dass sie es gut fand, dass es schrecklich zu lesen wäre, aber dass es ging. Ich sagte, dass ich frustriert gewesen war, aber dass ich das nun nicht mehr bin. Sie sagte: Leb wohl, Romantik.“ [Meine Übersetzung, S.K.]

13 „Was sollen wir nun tun, Karl Ove?“ [Meine Übersetzung, S.K.] 
traff jeg Linda, og solen gikk opp. Annen måte kann jeg ikke si det på. Solen gikk opp i livet mitt.“ (Knausgård 2009, 168) ${ }^{14}$ Tonje Aursland war zutiefst betroffen. Sie wusste nichts von diesen damaligen Gefühlen ihres Ex-Mannes. Was sie allerdings noch mehr verletzte, war Knausgårds Entschluss, sie einerseits zu einer Romanfigur zu machen, andererseits aber von ihr zu verlangen, das Buch nicht zu lesen. Das war die schlechteste Entscheidung, die er hatte treffen können, meinte sie (vgl. Aursland 2010).

\section{Enden der Autofiktion}

Knausgård versucht alles niederzuschreiben, woran er sich erinnert, und obwohl er bei Veröffentlichung des sechsten Bandes nur 43 Jahre alt war, könnte der Text potentiell unendlich sein. Auch wenn er erst nach 3600 Seiten den Schlusspunkt setzt, stellt sich die Frage, warum jetzt, warum schreibt er nicht weiter? Die Biographie endet mit dem Tod, doch wo endet die Autobiographie, vor allem die Autobiographie eines verhältnismäßig jungen Mannes, der zu Beginn dieses Projektes gerade mal zwei mäßig erfolgreiche Romane veröffentlicht hatte? Knausgård erklärt den Originaltitel Min Kamp im zweiten Band kurz:

Dagliglivet, med sine plikter og rutiner, var noe jeg utholdt, ingenting jeg gledet meg over, ingenting som ga meg mening eller gjorde meg lykkelig. Dette handle tikke om mangelen på lyst til å vaske gulv eller skifte bleier, men om noe mer grunnleggende, at jeg ikke opplevde verdien i det nære livet, men alltid lengtet bort derfra, og alltid hadde gjort det. Så livet jeg levde, var ikke mitt eget. Jeg forsøkte å gjøre det til mitt, det var den kampen jeg førte [Hervorhebung von mir, S.K.], for jeg ville jo det, men jeg mislyktes, lengselen etter noe annet hulte fullstendig ut alt det jeg gjorde. (Knausgård 2009, 67) ${ }^{15}$

14 „Unterhaltungen mit Tonje wurden seltener und seltener. Dann traf ich Linda und die Sonne ging auf. Anders kann ich das nicht ausdrücken. Die Sonne ging in meinem Leben auf.“ [Meine Übersetzung, S.K.]

15 „Der Alltag, mit seinen Pflichten und Routinen, war etwas, das ich aushielt, nichts, über das ich mich freute, nichts, was mir Sinn gab oder mich glücklich machte. Das hatte nichts mit einem Mangel an Lust, den Boden zu waschen oder Windeln zu wechseln, zu tun, sondern mit etwas Grundlegenderem, ich empfand nicht den Wert des Lebens meines Alltags, sondern strebte immer weg davon, und hatte dies schon immer getan. Das Leben, das ich lebte, war also nicht mein eigenes. Ich versuchte, es meines zu machen, das war der Kampf, den ich führte [Hervorhebung von mir, S.K.], denn natürlich wollte ich es, aber ich scheiterte, die Sehnsucht nach etwas Anderem erfüllte vollständig alles, was ich tat.“ [Meine Übersetzung, S.K.] 
Dieses Streben nach „etwas Anderem“ war das Streben nach Literatur. Sein Kampf ist also ein Kampf darum, sein Leben als Familienvater und Ehemann zu akzeptieren. Nur wenn er diesen Kampf gewinnt, kann er sein Projekt abschließen. Und genau das geschieht: Der 1117 Seiten starke sechste Band, in dem er einen 400 Seiten langen Essay über Adolf Hitlers Autobiographie Mein Kampf einfügte, endet mit dem schönen Satz: „Så skal vi ta toget hit til Malmö, så skal vi sette oss i bilen og så skal vi kjøre hjem til huset vårt, og hele veien skal jeg nyte, virkelig nyte tanken på at jeg ikke lenger er forfatter.“ (Knausgård 2011, 1116) ${ }^{16}$

Was darauf folgt, ist die Widmung, die nach diesem sechsbändigen Kampf eine der größten Liebeserklärungen der Weltliteratur darstellt, wenngleich Knausgård auch hier keine überraschenden Bilder oder Wendungen einfallen, sondern nur die berüchtigten drei Worte. „Til Linda, Vanja, Heidi og John. Jeg elsker dere“ (Knausgård 2011, 1117). ${ }^{17}$ Min Kamp ist also ein immenser Lord Chandos-Brief, der Knausgårds literarische Ambitionen beendet. Die Autobiographie endet nicht mit dem Tod, sondern mit dem Tod des Autors.

Das Schreckliche an Hitlers Autobiographie ist, dass sein Text Wahrheit geworden ist. Alles dort von ihm Fantasierte und Angekündigte hat er verwirklicht. Wäre das nicht der Fall gewesen, wäre seine Schrift eine obskure Anekdote der politischen Bewegungen der 1920er Jahre geblieben, die heutzutage nur spezialisierte Historiker ab und zu aus den Bibliotheken ausheben ließen. Knausgårds Kampf ist ein umgekehrter: Er möchte nicht sein Buch Wirklichkeit werden lassen, sondern die Wirklichkeit Literatur. Seine Autobiographie soll nicht den Weg vom Text in die Realität finden, um dort dann das Menschliche auszurotten, sondern soll auf Papier gebannt werden, um ihn endlich zum Menschen werden zu lassen. Er möchte Ehemann und Vater sein und nicht Schriftsteller, er möchte mehr Erfüllung im Familienleben finden als im Schreiben seiner Bücher. Also schreibt er sechs Bände darüber, wie schwer es für ihn ist, Familienmensch zu sein; wie hart es ist, sein Schreiben dem sogenannten wahren Leben unterordnen zu müssen. Er schreibt so lange darüber, bis er sich ausgeschrieben hat, bis nichts mehr übrig ist von seinem Schriftstellerdasein und nur noch der Mensch Knausgård zurückbleibt, als der er ganz am Schluss mit reinem Gewissen „Ich liebe Euch“ zu seiner Familie sagen kann.

16 „So werden wir den Zug zurück nach Malmö nehmen, dann werden wir uns ins Auto setzen und dann werden wir heimfahren zu unserem Haus, und den ganzen Weg lang werde ich den Gedanken genießen, wirklich genießen, dass ich nicht länger Schriftsteller bin.“ [Meine Übersetzung, S.K.]

17 „Für Linda, Vanja, Heidi und John. Ich liebe euch.“ [Meine Übersetzung, S.K.] 
Knausgård opfert also sein Schreiben der Liebe. Kermani deutet diese Option auch an. Wie immer unterscheidet er korrekt zwischen realem und fiktivem Autor: ${ }^{18}$

Anders als der Romanschreiber hat Navid Kermani auch gelernt, daß er nicht über Leichen gehen möchte, um an den flapsigen Ausdruck zu erinnern, der zweimal fiel, vielmehr dem Leben den Vorzug über die Kunst gibt, dem Jetzt, soweit es ihm eben zuteil wird, über die Ewigkeit, soweit sie einem Roman zuteil werden könnte, den ich schreibe. (Kermani 2011, 1217)

Kermani - wenn das die real lebende Schriftstellerpersönlichkeit ist - würde also auch das Leben über die Kunst stellen. Der fiktive Autor geht den umgekehrten Weg, auf Seite 1212 berichtet er, wie er sich am Mittwoch, den 15. Dezember 2010, in einer gerade einmal sechs Minuten langen Sitzung vor Gericht von seiner Frau, die er im Text lange Zeit immer nur, trotz aller Ehekrisen, „die Frau, die ich liebe“ nannte, scheiden lässt. Die Ehe ist gescheitert, die Liebe dahin. Damit kann sein Leben als Schriftsteller weitergehen. Sein autobiographischer Roman Dein Name endet allerdings nicht mit der Scheidung, sondern geht noch 17 Seiten weiter. Wo setzt Kermani also den Schlusspunkt in seiner „Selbstlebensbeschreibung“? „Das Totenbuch, da es die Vollständigkeit versuchen muß, endet erst mit dem eigenen Tod“ (Kermani 2015, pos. 13), stellt Navid Kermani bei einer Preisrede für seinen Roman Dein Name fest. Wie aber endet sein 1229-seitiger Versuch, sein Leben zwischen Donnerstag, den 8. Juni 2006, 11:18, und Samstag, den 11. Juni 2011, um 10:15, festzuhalten tatsächlich, da er ja noch lebt? Zehn Seiten vor Schluss schreibt Kermani, dass ihm sein Lektor nahelegt, den Roman in Isfahan, der Geburtsstadt seines Großvaters, dessen Biographie er unter anderem in Dein Name aufarbeitet, zu beenden. Kermani zweifelt, dass es möglich ist, in einem autobiographischen Text so einen Bogen zu spannen, „wenn wir doch alle höheren Gewalten ausgesetzt sind.“ (Kermani 2011, 1219) Er fliegt stattdessen nach Amerika und sein Verleger teilt ihm mit, dass ihm zehn zusätzliche Seiten bewilligt wurden, 24.000 Anschläge. Diese, und der Akkustand seines Laptops, bilden nun den Countdown zum Ende hin. Alle Themen des Romans werden noch einmal kursorisch berührt, die Anschläge immer weniger, bis zum Schluss nur noch 526 übrig sind. Der letzte Satz lautet: „Die Setzerin erinnert daran, daß jeder Absatzwechsel umgerechnet sechzig Anschläge kostet.“(Kermani 2011, 1229) Kermani endet also mit dem Topos

18 Kermani ärgert sich zu Recht, wenn man diese Trennung nicht vollzieht: „Ich bin nicht, das sage ich Dir offen, bei den Passagen verweilt, die Dein häusliches Leben schildern [es ist nicht mein häusliches Leben! ärgert sich der jüngere Kollege über die Gedankenlosigkeit selbst bei einem so berühmten Schriftsteller].“ (Kermani 2011, 1181). 
des zu Ende gehenden Papiers, der Kerze, die abgebrannt ist, und damit indirekt auch mit dem Ende allen Schreibens. Ohne Licht, Papier oder Strom, ohne Platz für Zeichen kann es auch keine Literatur mehr geben. Das Ende ist erreicht, auch wenn es zufällig ist, doch das ist der Schlusspunkt jeder Biographie, das ist jeder Tod. Dass sein Roman tatsächlich mit dem Schlusspunkt zu Ende gegangen war, musste Kermani erfahren, als er versuchte, ihn weiterzuschreiben. Er berichtet: „Der Roman ist zu Ende; mehrfach habe ich Anlauf genommen, ihn fortzuschreiben, nur um einsehen zu müssen, daß ich mich in Wiederholungen verfange; was zu erzählen war, scheint gerade in seiner Unvollständigkeit erzählt zu sein.“ (Kermani 2015, pos. 6)

Beide hier vorgestellten literarischen Großprojekte suchen auf ihre eigene Art nach einem zeitgemäßen Realismus. Die so fragmentierten Lebens- und Denkmodelle des 21. Jahrhunderts verlangen nach einer adäquaten Erzählform, für die sich anscheinend die direkte Auseinandersetzung mit dem erzählenden Individuum, als Meta-Autofiktion, am besten eignet.

Knausgård hat seit seinem Bekenntnis, kein Autor mehr zu sein, vier weitere als Roman herausgegebene Bücher geschrieben, in denen er seinen inzwischen vier Kindern das Leben auf verschiedene Weise vorstellt. Natürlich ist es keine Überraschung, dass er weiterschreibt, doch bleibt es spannend, in welche Richtung er sich als Schriftsteller weiterentwickeln wird. Vielleicht nimmt er einmal ein Kapitel aus Min Kamp und macht daraus einen eigenen Roman. Genau das hat Navid Kermani bereits getan. Große Liebe ist der Roman seiner ersten Liebe, die in Dein Name auf ein paar Seiten behandelt wird.

„Mein Leben kann nur ich beschreiben, weil ich das Innere gebe; das von Göthe hätte ein Nebenherläufer beobachten und also mittheilen können“ (nach: Kermani 2011, 1123), ${ }^{19}$ schreibt Jean Paul. Goethe schrieb sein individuelles Leben auf, Jean Paul eines, in dem alle Leben sich wiederfinden. „Das Ich gilt, aber nicht mein Ich“ (nach: Kermani 2011, 1123), fasst er zusammen. Kermani nimmt diesen Gedanken auf und erzählt auf der ersten Seite von Große Liebe, dem Nachfolgeroman von Dein Name, die Anekdote eines Königs, der spöttisch einem Bettler zuruft: „Du würdest wohl gern ich sein“, worauf dieser antwortete, „Nein, ich möchte nicht ich sein.“ (Kermani 2014, 1) Er ist aber ein Ich - und damit ein Du, und damit spricht ihn sowohl Min Kamp als auch Dein Name direkt an: Er muss seinen Kampf kämpfen, um am Ende seinen Namen in das große Totenbuch des Lebens eintragen zu können.

19 Jean Pauls unvollendet gebliebene Selberlebensbeschreibung ist ein gewitzter Vorgänger heutiger Autofiktionen, dafür sorgt schon allein Jean Pauls „Unlust, ohne Fiktion über sich selbst sprechen zu müssen.“ (Wuthenow 2013, 164) 


\section{Literaturverzeichnis}

Aursland, Tonje. Tonjes versjon - ein radiodokumentar om å bli ufrivillig romanfigur. Radiodokumentation: NRK, 02.10.2010, 46min $23 \mathrm{sec}$.

Darrieussecq, Marie. „L’autofiction, un genre pas sérieux“. Poètique 107 (1996): 367-380.

Doubrovsky, Serge. Fils. Paris: Éditions Galilée, 1977.

Egeland, Marianne. „Recognition and Authenticity in Life Writing“. The International Journal of the Book 13.4 (2015): 11-22.

Fliedl, Konstanze. „Deutung und Diskretion. Zum Problem des Biographismus im Fall Bachmann - Frisch“. Revista de Filología Alemana 10 (2002): 55-70.

Hoffmann, Torsten. „Trennungsprobleme. Navid Kermanis Autofiktionen“. Text und Kritik 217 (2018) (Navid Kermani): 14-22.

Keil, Frank. „Das eigene Leben als Textprojekt. Navid Kermani liest im Literaturhaus quer“. Die Welt, 21.10.2011.

Kermani, Navid. Dein Name. München: Hanser, 2011.

Kermani, Navid. „Rede zur Verleihung des Kleist-Preises 2012“. Kleist-Jahrbuch 2013. Hg. G. Blamberger, I. Breuer, W. de Bruyn und K. Müller-Salget. Stuttgart: J. B. Metzler, 2013. 13-20.

Kermani, Navid. Große Liebe. München: Hanser, 2014.

Kermani, Navid. „Jeder Mensch eine Menschheit: Dankrede zum Joseph-Breitbach-Preis für den Roman Dein Name“. München: Hanser Box (E-book), 2015.

Kermani, Navid. Sozusagen Paris. München: Hanser, 2016.

Kermani, Navid. „Brief an Elliot Robertson, den Manager von Neil Young“. Text und Kritik 217 (2018) (Navid Kermani): 40-42.

Knausgård, Karl Ove. Min kamp. Roman: II. Oslo: Forlaget Oktober, 2009.

Knausgård, Karl Ove. Min kamp. Roman: V. Oslo: Forlaget Oktober, 2010.

Knausgård, Karl Ove. Min kamp. Roman: Vl. Oslo: Forlaget Oktober, 2011.

Kutzenberger, Stefan. „Autofiction and Its (Involuntary) Protagonists: A Comparison of Autofictional Novels by Mario Vargas Llosa, Javier Cercas, Karl Ove Knausgård, and Navid Kermani“. Taking Stock - Twenty-Five Years of Comparative Literary Research. Internationale Forschungen zur Allgemeinen und Vergleichenden Literaturwissenschaft 200. Hg. Norbert Bachleitner, Achim Hölter und John A. McCarthy. Leiden und Boston: Brill, 2019. 397-420.

Luserke-Jaqui, Matthias. Kleine Literaturgeschichte der großen Liebe. Darmstadt: Wissenschaftliche Buchgesellschaft, 2011.

Ott, Christine, und Weiser, Jutta. „Autofiktion und Medienrealität. Einleitung“. Autofiktion und Medienrealität. Kulturelle Formungen des postmodernen Subjekts. Hg. Christine Ott und Jutta Weiser. Heidelberg: Winter, 2013. 7-18.

Shields, David. Reality Hunger: A Manifesto. New York: Knopf, 2010.

Wittgenstein, Ludwig. Bemerkungen über die Farben. Über Gewißheit. Zettel. Vermischte Bemerkungen. Werkausgabe in 8 Bänden: Band 8. Frankfurt/M.: Suhrkamp, 2015.

Wuthenow, Ralph-Rainer. „Gegenwärtige Vergangenheit und erinnerte Zukunft“. Nachwort zu: Jean Paul. Selberlebensbeschreibung. Konjektural-Biographie. Stuttgart: Reclam, 2013. 157-180.

Zipfel, Frank. „Autofiktion. Zwischen den Grenzen von Faktualität, Fiktionalität und Literarität?“ Grenzen der Literatur. Zu Begriff und Phänomen des Literarischen. Hg. Simone Winko, Fotis Jannidis und Gerhard Lauer. Berlin und New York: Walter de Gruyter, 2009. 285-314. 
Stefan Kutzenberger (geboren 1971 in Linz) lebt als Literaturwissenschaftler und Schriftsteller in Wien. Zahlreiche Publikationen und Präsentationen zur Visualisierung von Literatur, zur Intermedialität in Wien um 1900 und zur literarischen Wechselbeziehung zwischen der europäischen und der lateinamerikanischen Literatur. 